LA-UR- $08-8056$

Approved for public release;

distribution is unlimited.

Title: $\quad$ Reliable Estimation of Shock Position in Shock-Capturing Compressible Hydrodynamics Codes

\author{
Author(s): $\quad$ Eric M. Nelson \\ Intended for: $\quad$ AIAA Aerospace Sciences Meeting \\ 5-8 January 2009 \\ Orlando, FL
}

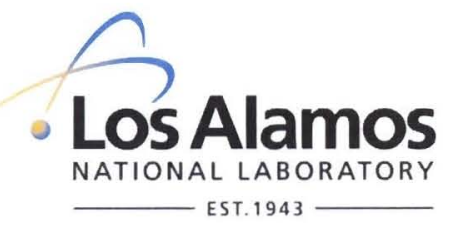

Los Alamos National Laboratory, an affirmative action/equal opportunity employer, is operated by the Los Alamos National Security, LLC for the National Nuclear Security Administration of the U.S. Department of Energy under contract DE-AC52-06NA25396. By acceptance of this article, the publisher recognizes that the U.S. Government retains a nonexclusive, royalty-free license to publish or reproduce the published form of this contribution, or to allow others to do so, for U.S. Government purposes. Los Alamos National Laboratory requests that the publisher identify this article as work performed under the auspices of the U.S. Department of Energy. Los Alamos National Laboratory strongly supports academic freedom and a researcher's right to publish; as an institution, however, the Laboratory does not endorse the viewpoint of a publication or guarantee its technical correctness. 


\title{
Reliable Estimation of Shock Position in Shock-Capturing Compressible Hydrodynamics Codes
}

\author{
Eric M. Nelson* \\ LANL, Los Alamos, NM 87545
}

\begin{abstract}
The displacement method for estimating shock position in a shock-capturing compressible hydrodynamics code is introduced. Common estimates use simulation data within the captured shock, but the displacement method uses data behind the shock, making the estimate consistent with and as reliable as estimates of material parameters obtained from averages or fits behind the shock. The displacement method is described in the context of a steady shock in a one-dimensional lagrangian hydrodynamics code, and demonstrated on a piston problem and a spherical blast wave. The displacement method's estimates of shock position are much better than common estimates in such applications.
\end{abstract}

\section{Introduction}

Common estimates of shock position in a shock-capturing compressible hydrodynamics code rely on snapshot data within the captured shock. The estimate may be the location at which a material parameter (e.g., velocity or density) crosses a specified fraction (typically half) of the jump across the shock, the location of the maximum slope in the material parameter, or the location of peak artificial viscous stress.

Such estimates vary anomalously by a substantial fraction of a zone size depending on the position of the captured shock relative to the mesh. Such unphysical variation is evident in the step by step estimated shock position as the captured shock propagates from one zone to the next. Computational physicists have thus observed that estimates of shock position are not very precise, being certain to a substantial fraction of a zone size at best.

Simple estimates of shock velocity based on successive common estimates of shock position are likewise uncertain, especially if adjacent time steps are employed.

Common estimates of material parameters are typically an average or fit to data outside the captured shock. Provided there are no comparable size waves or additional shocks nearby, estimates of material parameters before and after a steady or nearly steady shock can be very precise. Uncertainty in a nonsteady shock is mainly due to uncertainty in the shock position to which one extrapolates the fit.

In this paper I introduce the displacement method for estimating shock position and shock velocity as a reliable alternative to common estimates of shock position. Like common estimates of material parameters, the displacement method fits to data outside of the captured shock and thus avoids dependence on the profile of the captured shock, step by step variations of the profile, and the profile's dependence on the artificial viscosity treatment. The displacement method is likewise degraded by the presence of comparable size waves or additional shocks nearby.

For the purpose of clear and simple exposition, I describe and demonstrate the displacement method in the context of a steady shock in a one-dimensional compatible ${ }^{1}$ lagrangian hydrodynamics code. The method is easily adapted to two and three dimensional simulations, to compressible eulerian hydrodynamics codes, and to non-steady shocks. For non-steady shocks, I briefly discuss the adaptation and then demonstrate the method in a one-dimensional lagrangian simulation of a blast wave ${ }^{2}$. Adaptation to two and three dimensional simulations and to eulerian codes are only briefly discussed.

The demonstrations include comparison with analytic solutions, comparison with the common estimates of shock position, an argument for why the displacement method is the most accurate as well as the most precise estimate of shock position, and demonstration of improved precision with increasing number of points in the fit.

I intend to use the displacement method in verification studies, but the method should also be useful wherever reliable estimates of shock position or shock velocity are desired from compressible hydrodynamic

* Technical Staff Member, Computational Analysis and Simulation Group, MS F644. 


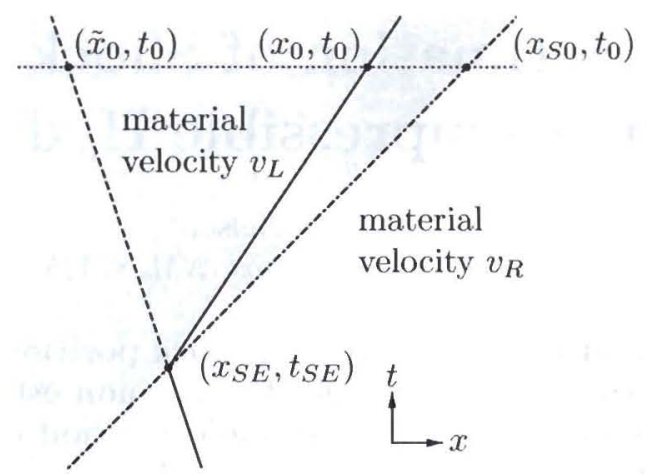

Figure 1. Space-time diagram of a steady shock (dot-dash line), a particle trajectory (two solid line segments), the corresponding unshocked particle trajectory (dashed line), and the time $t_{0}$ of a snapshot (dotted line). The shocked material (shaded) has material velocity $v_{L}$, the undisturbed material (not shaded) has material velocity $v_{R}$. This particular diagram is the piston problem observed in a frame moving to the right with velocity $1 / 3$, hence $v_{R}=-1 / 3, v_{L}=2 / 3$ and shock velocity $v_{S}=1$.

simulations, such as fitting equation of state shock experiments for material-specific model parameters. In simulations where resolution of shock position is the primary driver for a fine mesh, the displacement method may make a coarser mesh viable.

\section{The Displacement Method for a Steady Shock}

Consider a steady shock with shock velocity $v_{S}$ and shock position $x_{S}=v_{S}\left(t-t_{0}\right)+x_{S 0}$ propagating from shocked material on the left, $x<x_{S}$, into undisturbed material on the right, $x>x_{S}$. See figure 1 . The material velocity before the shock (on the right) is $v_{R}$, and the material velocity after the shock (on the left) is $v_{L}$. A particle at position $x_{0}$ behind the shock at time $t_{0}$ is following the trajectory $x=v_{L}\left(t-t_{0}\right)+x_{0}$ ever since it encountered the shock at time

$$
t_{S E}=t_{0}-\frac{x_{S 0}-x_{0}}{v_{S}-v_{L}}
$$

and position

$$
x_{S E}=v_{S}\left(t_{S E}-t_{0}\right)+x_{S 0}=v_{L}\left(t_{S E}-t_{0}\right)+x_{0}=\frac{v_{S} x_{0}-v_{L} x_{S 0}}{v_{S}-v_{L}} .
$$

Had the particle not encountered the shock, the particle's position at time $t_{0}$ would be

$$
\tilde{x}_{0}=v_{R}\left(t_{0}-t_{S E}\right)+x_{S E}=\frac{\left(v_{S}-v_{R}\right) x_{0}-\left(v_{L}-v_{R}\right) x_{S 0}}{v_{S}-v_{L}} .
$$

Call $\tilde{x}_{0}$ the unshocked position of the particle. The displacement of the particle due to encountering the shock is

$$
x_{0}-\tilde{x}_{0}=\left(v_{L}-v_{R}\right)\left(t_{0}-t_{S E}\right)=\frac{v_{L}-v_{R}}{v_{S}-v_{L}}\left(x_{S 0}-x_{0}\right)=\frac{v_{L}-v_{R}}{v_{S}-v_{R}}\left(x_{S 0}-\tilde{x}_{0}\right) .
$$

Call $x_{0}-\tilde{x}_{0}$ the shock-induced displacement of the particle.

In a snapshot of a lagrangian hydrodynamic simulation, the position of the $i$ th node is $x_{i}$. The unshocked positions $\tilde{x}_{i}$ of each node can often be obtained from the initial conditions specified for a problem. In some cases the unshocked positions may be extrapolated or computed from a snapshot taken at an earlier time.

For a steady shock, the displacement $x_{i}-\tilde{x}_{i}$ depends linearly on position $x_{i}$, so one fits a line to select nodes behind the shock. The intercept with the horizontal axis (zero shock-induced displacement) gives a precise and reliable estimate of the shock position. The slope $-\left(v_{L}-v_{R}\right) /\left(v_{S}-v_{L}\right)$, combined with estimates for the material velocities $v_{L}$ and $v_{R}$ on either side of the shock, gives a precise and reliable estimate for the shock velocity $v_{S}$. Reliability will be discussed and demonstrated later. 

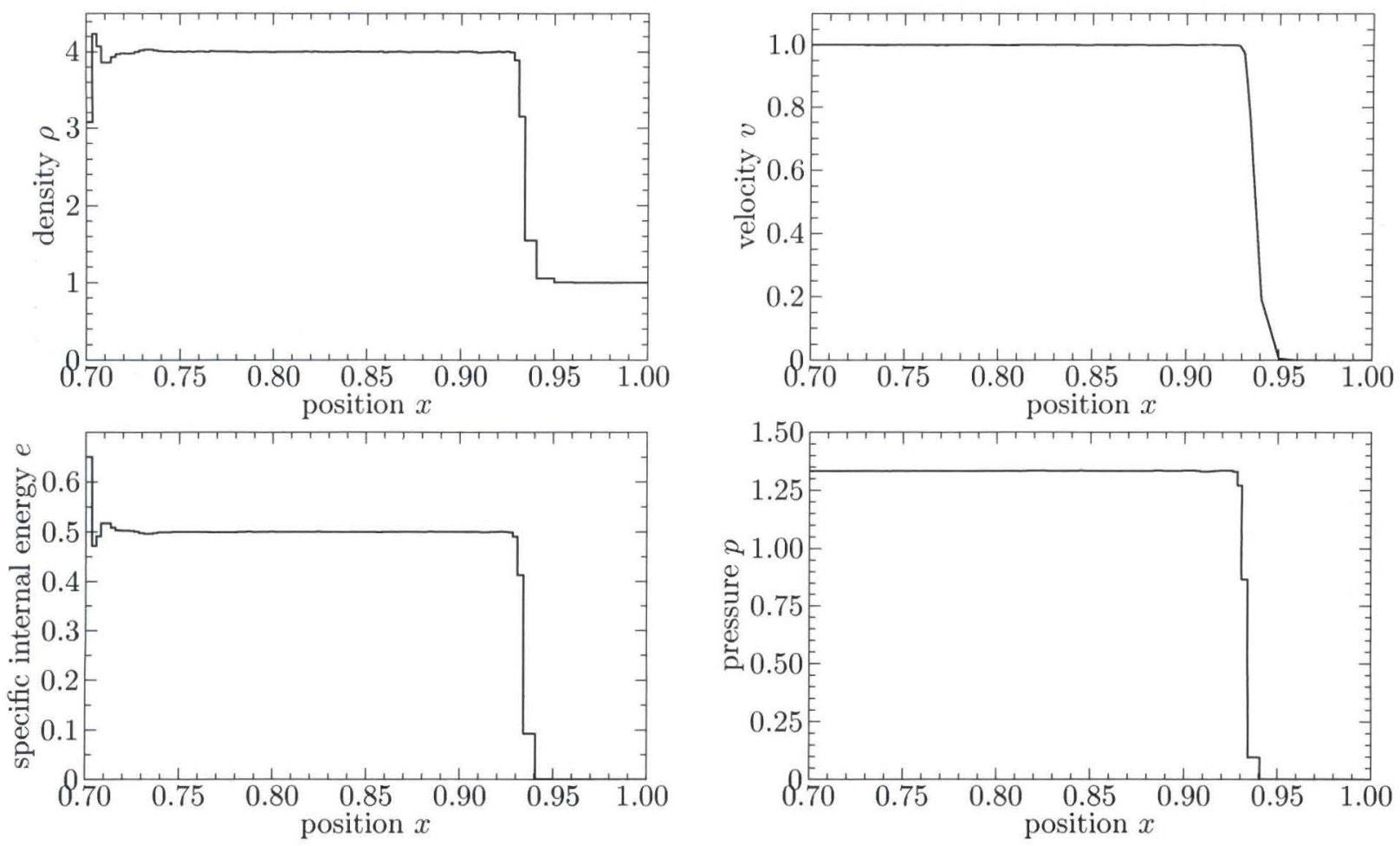

Figure 2. Density, velocity, specific internal energy and pressure at $t=0.7$ of the piston problem.

As an example, consider the piston problem. A piston on the left with unit velocity shock compresses an ideal gas, $\gamma=5 / 3$, that is initially unit density, cold, and at rest. Figure 2 shows typical snapshots of material parameters at $t=0.7$. The simulation has 100 zones initially distributed uniformly over $0 \leq x \leq 1$.

Figure 3 shows shock-induced displacement versus position $x$ or unshocked position $\tilde{x}$ in the piston problem. The unshocked positions are just the initial positions of each node. In both cases, the nodes behind the shock are close to a line, and in both cases a simple least squares fit to nodes 82 through 90 gives shock position $x_{S 0}=0.934420$, which is slightly ahead of the theoretical shock position $x_{S 0}=0.933333$.

In problems where the unshocked positions $\tilde{x}_{i}$ are given exactly by specified initial conditions, one might prefer to fit the displacement versus unshocked position $\tilde{x}$ so that computational errors in the positions $x_{i}$ are not introduced twice into the fit. In problems where the unshocked positions $\tilde{x}_{i}$ are not so well known, such as a noisy extrapolation from an earlier snapshot, one might prefer instead to fit the displacement versus position $x$ so that the larger errors in $\tilde{x}_{i}$ are not introduced twice into the fit.

\section{Consistency of the Displacement Method with Common Estimates of Material Velocity}

The displacement method for estimating shock position and shock velocity is consistent with the common estimates for material velocity in the following sense. Both estimates require a choice of nodes (or zones) and a choice of weighting scheme to perform the fit or average. The same choices can be made for both estimates, making the two estimates consistent.

The shock position estimated by the displacement method is an extrapolation of the fit, which contrasts with the estimates of material parameters obtained by averaging. But the shock position nonetheless behaves like the material velocity estimate in two ways: (1) the estimate depend on the selection of nodes for the average or fit, and (2) the precision or reliability of the estimate improves as the number of nodes in the average or fit is increased.

Both aspects can be observed in the time evolution of the estimates. For my demonstration, the zones for the estimate are selected as follows for each time step. Identify the nominal shock front as the zone with greatest $|d v / d r|$. Let the index of this zone be $j_{S}$. Average over, or fit to, zones $j_{S}-5-n_{z} \leq j \leq j_{S}-5$. The estimate excludes the four zones behind the nominal shock front. The selection of zones for the estimate 

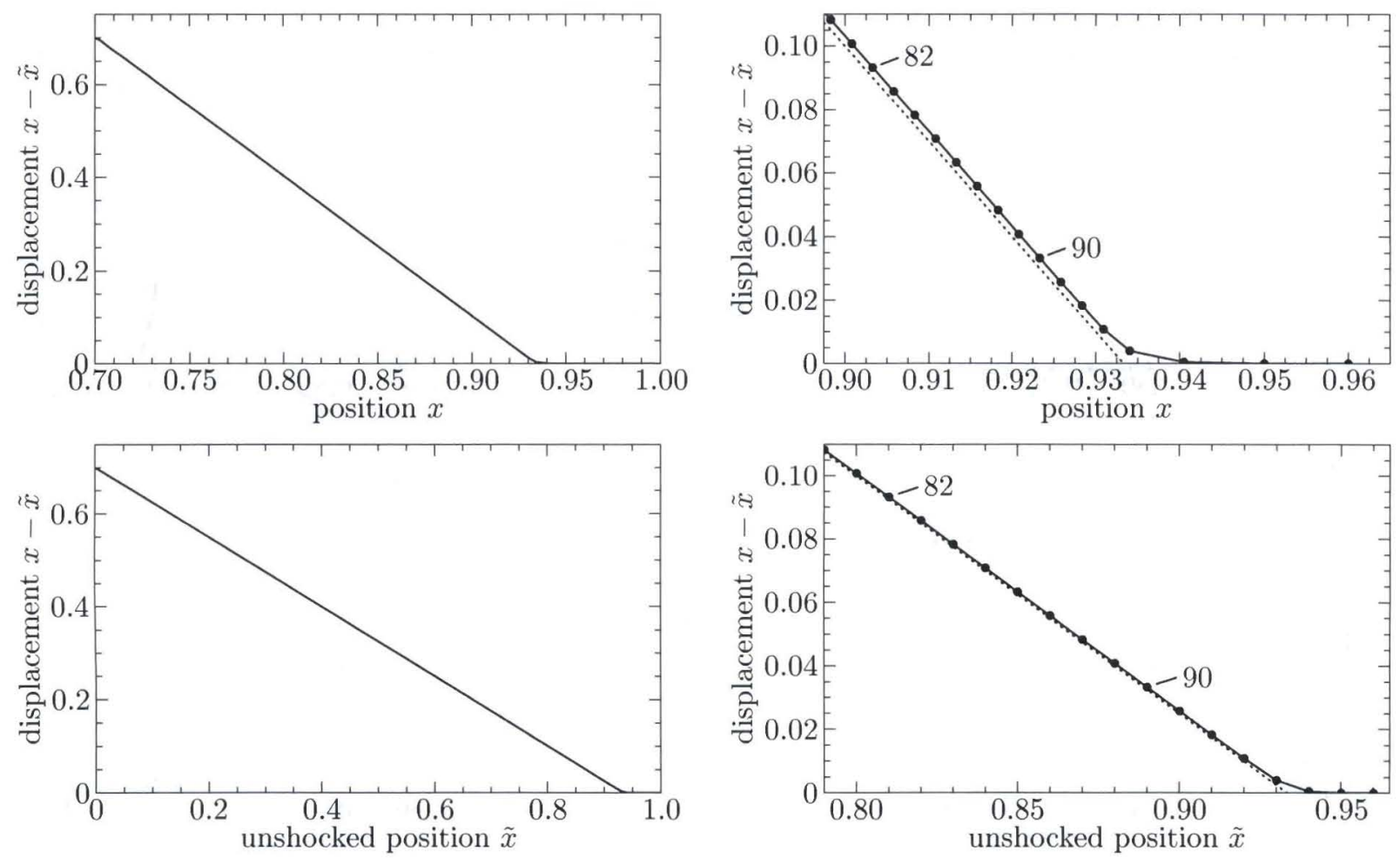

Figure 3. Node displacement due solely to the shock, $x-\tilde{x}$, plotted as a function of position $x$ (upper plots) or unshocked position $\tilde{x}$ (lower plots). Left plots shows the entire model, right plots shows the captured shock and the region just behind the shock. Dotted curve is the theoretical shock, not a fit to simulation data. Nodes 82 through 90 are identified for the fit described in the text.

will change when the nominal shock front propagates from one zone to the zone's neighbor on its right: $j_{S}\left(t_{i+1}\right)=j_{S}\left(t_{i}\right)+1$.

Figure 4 shows how estimates of the shocked material velocity $v_{L}$ evolves in time near $t=0.7$. Four estimates, each with a different number of zones $n_{z}$ included in the average, are shown. The estimates evolve smoothly in time when the zones in the estimate are fixed, indicated by points connected with solid line segments. The estimates exhibit some sudden jumps when the zones selected for the estimate change, indicated by pairs of points connected with dotted line segments.

Figure 4 also shows how the estimates of shock position and shock velocity, computed with the displacement method, evolve in time. The analytic shock position is subtracted from the estimate to better show the variation. The estimate of shock position is not as smooth as the estimate of material velocity, but some jumps are still noticeable when the zones selected for the fit change. The estimate of shock velocity is nearly as smooth as material velocity, but some small kinks are evident.

The figure shows that the overall variation of the estimates decrease as the number of zones in the estimates increase. Taking the range of variation (maximum minus minimum) in the time range as an approximate measure of an estimate's precision, one finds that the precision is roughly inversely proportional to the number of zones selected for the estimate, as shown in figure 5. The nodes of the simulation are sufficiently correlated in space and time to make the precision of the estimates scale like $1 / n_{n}$ rather than the $1 / \sqrt{n_{n}}$ scaling one would obtain if the samples were statistically independent.

I deliberately limited figure 5 to $n_{z} \leq 50$. The shock position's range of variation begins to increase rather than decrease as I average over much more than 50 nodes. The increased variation is due to the fit encountering a larger (and persistent) variation of displacement correlated over a longer spatial scale starting near node 40 and extending to the left toward the piston. See figure 6 . The fit is encountering the effects of wall heating. The displacement method's ability to observe such remnants of wall heating nearly 40 zones from the wall is a testament to the method's precision. 

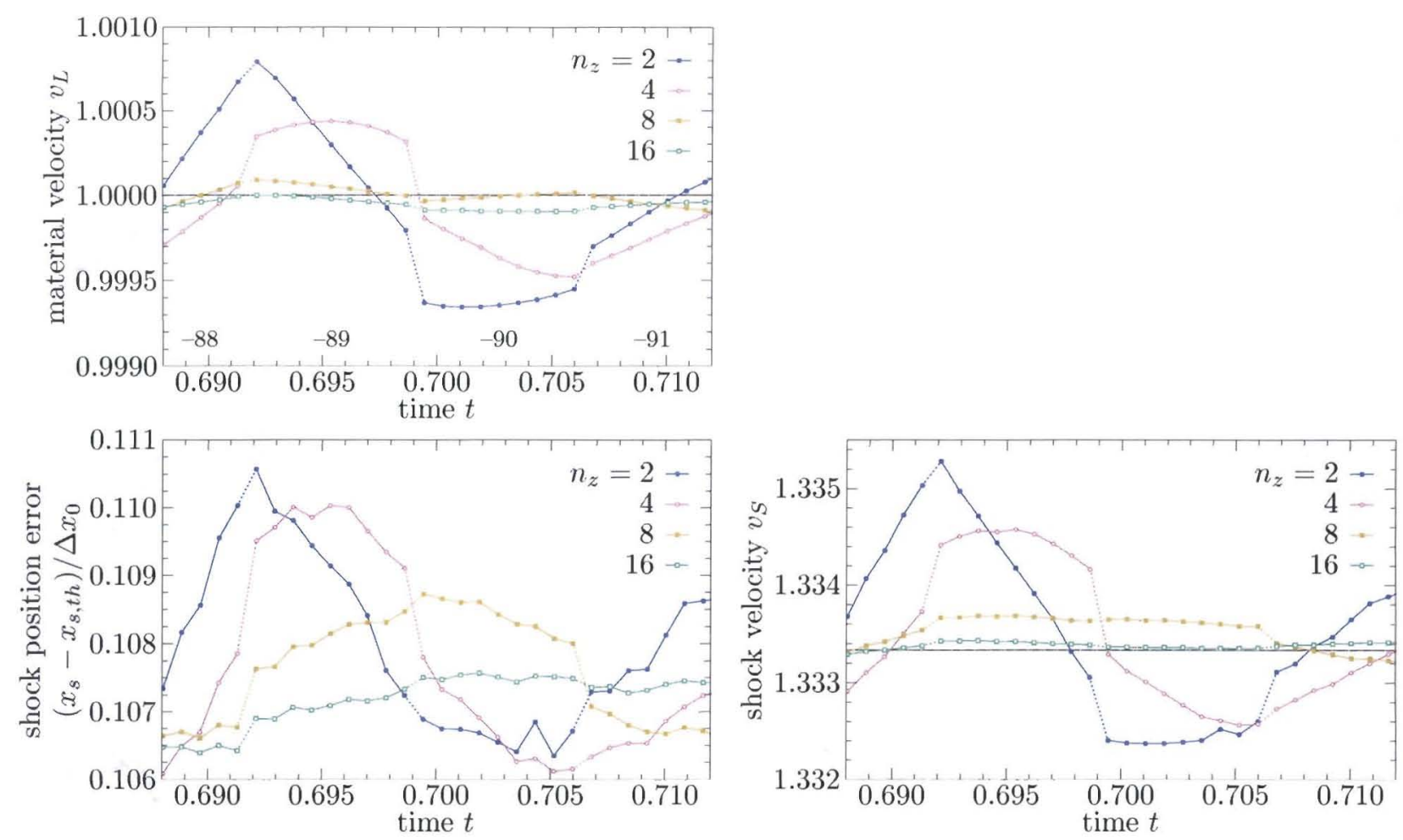

Figure 4. Material velocity, shock position error relative to initial zone size, and shock velocity estimated from average or fit to $n_{z}+1$ nodes, as the simulation evolves in time near $t=0.7$, and for various number of zones $n_{z}$. Dotted portion of curves show where the nodes selected for averaging shift one node to the right. The index (preceded by a dash) of the rightmost node included in the average or fit is listed along the bottom of the material velocity plot. Black horizontal line is the theoretical material velocity or shock velocity.

\section{Common Practice for Estimating Shock Position}

Common estimates of shock position contrast starkly with common estimates of material parameters in the following way. The shock position is computed from simulation data within the captured shock, not simulation data behind the captured shock. The common estimates of shock position are considered reliable at best to a zone size, and the estimates may depend strongly on the shock capturing algorithm, such as the coefficients in the artificial viscosity calculation.

One common estimate of shock position is the place where a material parameter obtains a value that is the average of the material parameter before and after the shock. This is a three step process: first estimate the material parameter after the shock (and before if necessary), compute the average, then find the position within the captured shock where the material parameter obtains the average value. The material parameter may be interpolated linearly to obtain a subgrid-scale shock position. Call this the half method.

Another common estimate of shock position is the place where the spatial derivative of the material parameter obtains its maximum absolute value. The spatial derivative may be interpolated quadratically to obtain a subgrid-scale shock position. Call this the maximum derivative method. For interpolation, I center the derivative of zone-centered material parameters halfway between zone centers. The derivative of velocity is zone centered.

Yet another common estimate of shock position is the place where the artificial viscous stress, $q$, obtains its maximum value. The artificial viscous stress may be interpolated quadratically to obtain a subgrid-scale shock position. Call this the maximum $q$ method.

Figure 7 shows the captured shock in the piston problem, along with linear interpolations of the zonecentered material parameters. The dotted line is the theoretical shock. Table 1 lists the various estimates of the shock position, along with their error relative to the theoretical shock position. The right side of the table (labeled "nearest") lists estimates without interpolation: the estimates will be a zone center (for the 

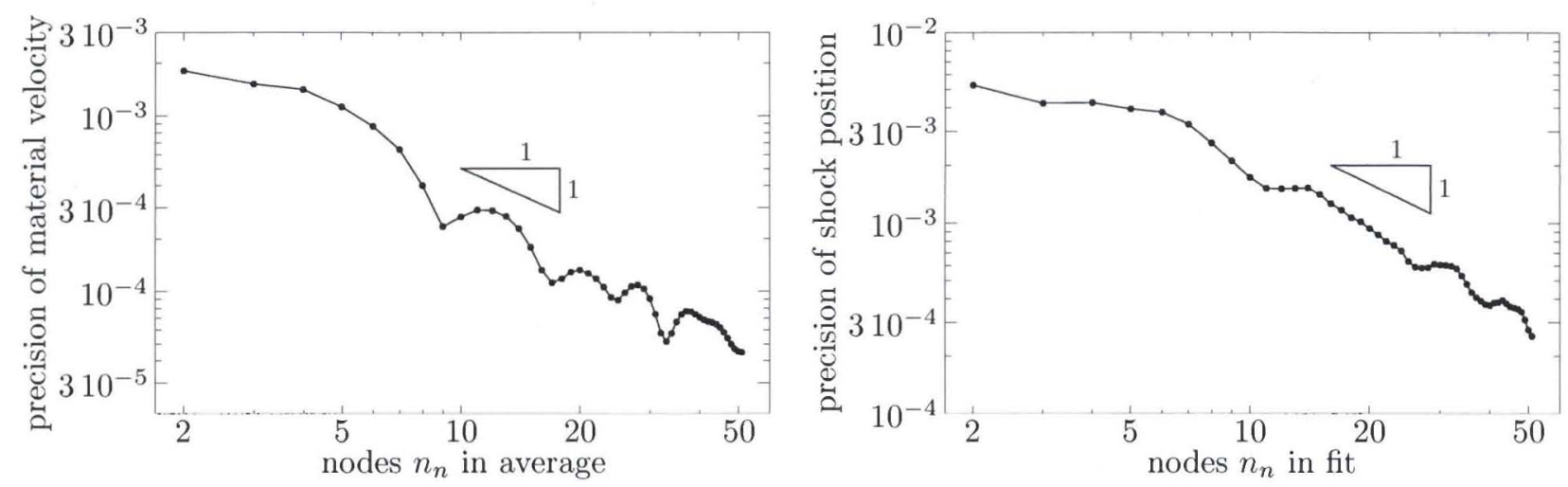

Figure 5. The variation ( $\max -\min$ ) of estimates for shocked material velocity (left plot) and shock position (right plot) in the time interval $0.688<t<0.712$, versus number of nodes $n_{n}=n_{z}+1$ in the straight average or fit. Triangles indicate slope -1 . The variation of both estimates is roughly inversely proportional to the number of nodes.
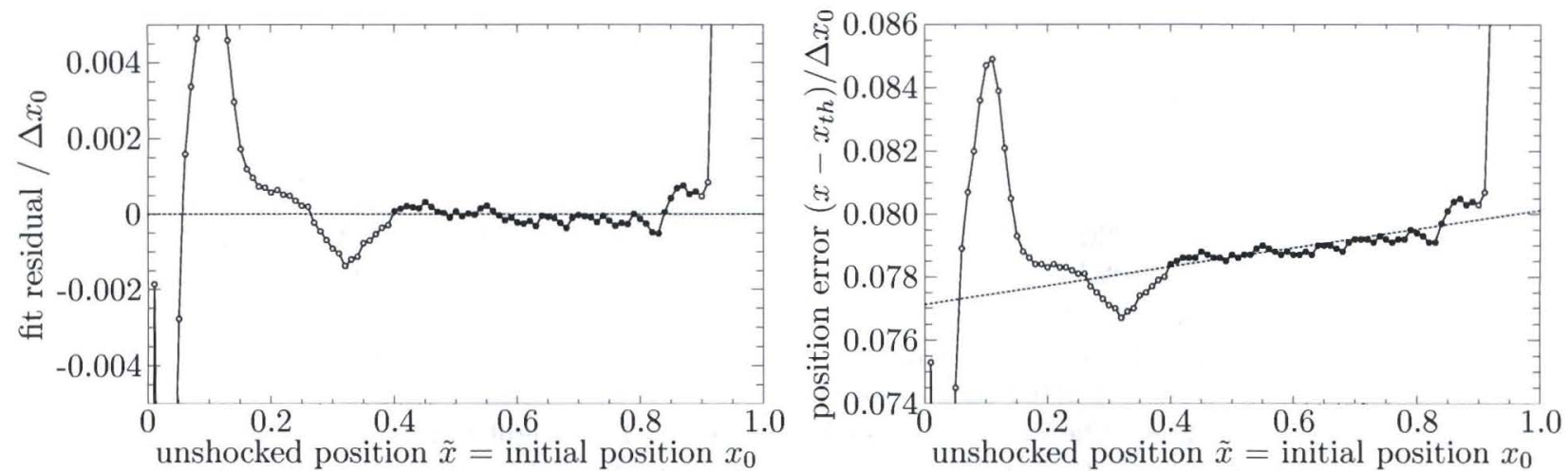

Figure 6. The deviation from the fit to shock-induced displacement, versus unshocked position. The solid circles, nodes 41 to 90 , are the nodes included in the fit for $n_{n}=n_{z}+1=50$, and at $t=0.7$. The open circles are nodes excluded from the fit. The large deviations on the left $(\tilde{x} \leq 0.4)$ are due to wall heating. The deviation on the right $(\tilde{x} \geq 0.9)$ is the captured shock. The right plot is the same data, but plotted as the deviation of node position from theory rather than deviation from the fit. The dotted line is the fit in both plots.

half $v$, max $|d v / d x|$ and $\max q$ methods) or a node (for the remaining common estimates).

The estimates differ by a substantial fraction of the local zone size. The size of the two zones in the middle of the captured shock are $0.646 \Delta x_{0}$ and $0.318 \Delta x_{0}$.

If agreement with theory were the sole criterion for choosing an estimate, then the table suggests the estimates computed from pressure would be best, at least for this piston problem.

But the shock in a simulation will not necessarily agree with theory. The difference between simulation and theory is something I want to measure in test problems such as the piston problem. With this motive in mind, the comparisons with theory in table 1 make no effective distinction between the estimates.

The simulated node positions behind the shock front are roughly $0.08 \Delta x_{0}$ ahead of where they should be theoretically. The difference is mostly due to the density deficit at the piston that was induced by wall heating, as seen in the density plot in figure 2 and in the node position error in figure 6 . The advanced node positions corresponds to a simulated shock that is ahead of theory by about $0.11 \Delta x_{0}$, which is consistent with the displacement method. So the displacement method is giving a shock position whose error (relative to theory) can be explained by errant behavior (wall heating) elsewhere in the simulation.

A great distinction between the estimates is observed in their time evolution. Shock position is plotted versus time near $t=0.7$ in figure 8 for the displacement method and the common subgrid-scale (interpolated) estimates. The displacement method's estimate is a straight line (on this scale), as one would expect for the position of a steady shock. The other estimates show significant deviations from a straight line. The 

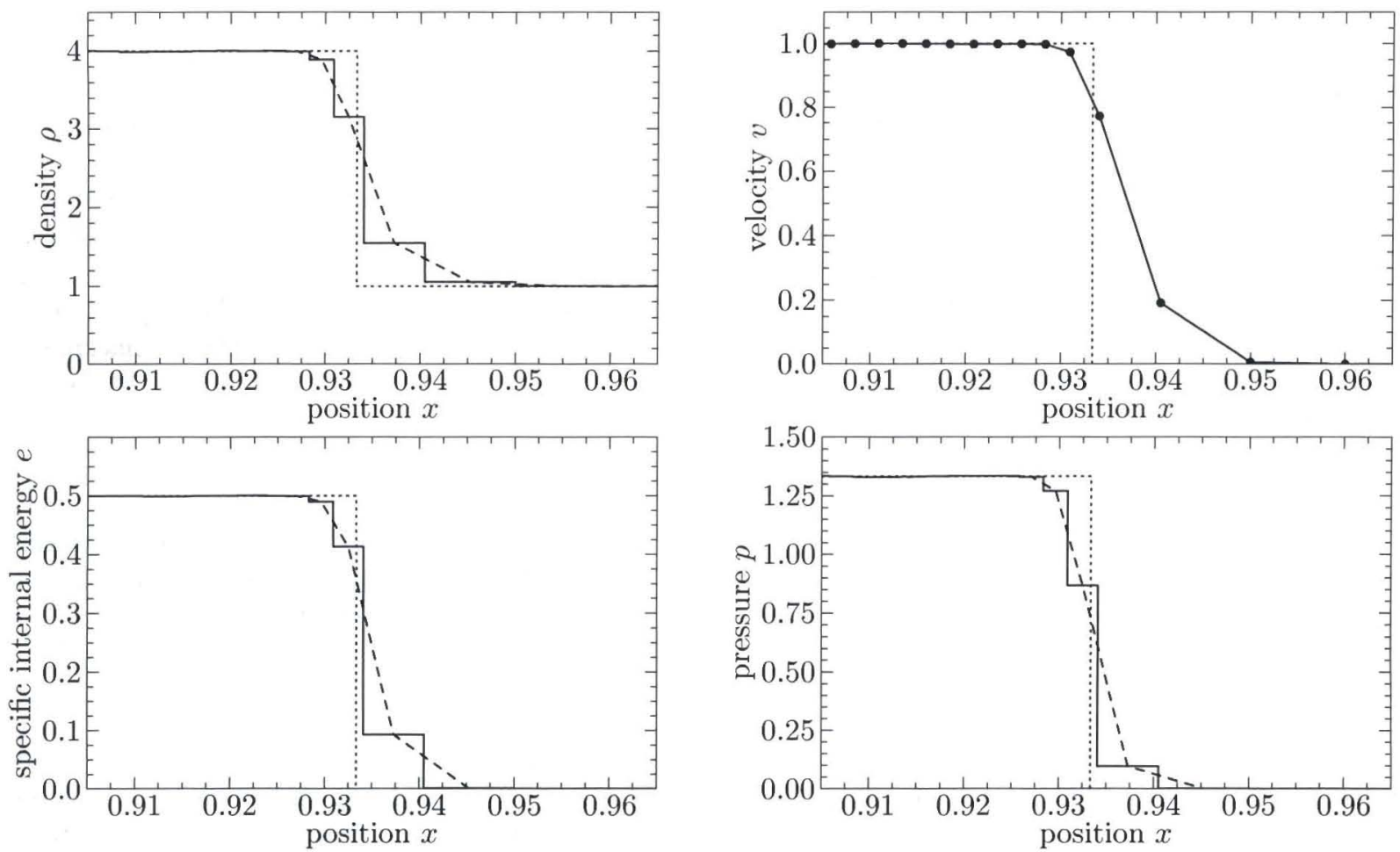

Figure 7. Density, velocity, specific internal energy and pressure at the captured shock. Dashed curves linearly interpolate zone-centered variables through the zone centers. Dotted curve is the theoretical shock.

\begin{tabular}{|r|l|l|l|l|}
\hline method & position & error & position & error \\
\hline theory & 0.933333 & - & & \\
displacement & 0.934420 & $0.109 \Delta x_{0}$ & \multicolumn{2}{|c|}{} \\
\cline { 2 - 5 } & \multicolumn{2}{|c|}{ interpolated } & \multicolumn{2}{|c|}{ nearest } \\
\cline { 2 - 5 }$\rho$ half & 0.934423 & $0.109 \Delta x_{0}$ & 0.934055 & $0.072 \Delta x_{0}$ \\
$e$ half & 0.934916 & $0.158 \Delta x_{0}$ & 0.934055 & $0.072 \Delta x_{0}$ \\
$p$ half & 0.933719 & $0.039 \Delta x_{0}$ & 0.934055 & $0.072 \Delta x_{0}$ \\
$v$ half & 0.937078 & $0.374 \Delta x_{0}$ & 0.937283 & $0.395 \Delta x_{0}$ \\
$|d \rho / d x| \max$ & 0.934563 & $0.123 \Delta x_{0}$ & 0.934055 & $0.072 \Delta x_{0}$ \\
$|d e / d x| \max$ & 0.935743 & $0.241 \Delta x_{0}$ & 0.934055 & $0.072 \Delta x_{0}$ \\
$|d p / d x| \max$ & 0.933868 & $0.053 \Delta x_{0}$ & 0.934055 & $0.072 \Delta x_{0}$ \\
$|d v / d x| \max$ & 0.937319 & $0.399 \Delta x_{0}$ & 0.937283 & $0.395 \Delta x_{0}$ \\
$q \max$ & 0.937117 & $0.378 \Delta x_{0}$ & 0.937283 & $0.395 \Delta x_{0}$ \\
\hline
\end{tabular}

Table 1. Estimates of shock position at $t=0.7$, and the corresponding error relative to the theoretical shock position. Common estimates based on material parameters in the captured shock are listed in the last 9 rows of the table. Subgrid position obtained with interpolation is on the left, nearest node or zone center (no interpolation) is on the right.

displacement method is much more reliable in this sense.

Figure 9 shows the error relative to the theoretical shock as a function of time, so that the behavior of the individual estimates can be observed. On this scale the displacement method exhibits no variation as the shock propagates from one zone to the next, while the common estimates exhibit substantial variation. The error nominally depends on the position of the shock relative to the grid, and so I call such artificial 
variations grid phase oscillations.

Among the common interpolated estimates, the half methods have roughly half the variation of the maximum derivative and maximum $q$ methods. The half $p$ method is most reliable (least variation from time step to time step), while the maximum $q$ method is the least reliable (most variation from time step to time step).

Figures 10 and 11 show the grid-scale (not interpolated) estimates, along with the displacement method.

In the half method, the shock position is where the material parameter has experienced half of the jump across the shock. A different fraction of the jump may be chosen, yielding a different estimate of the shock position. Increasing the fraction above one half reduces the variation of the shock position a little, as shown in figure 12, but there is no fraction that makes the estimate as reliable as the displacement method. An interpolation scheme much more sophisticated than linear interpolation can reduce the variation substantially.

\section{An Unsteady Shock: The Sedov Blast Wave Problem}

I have just introduced the displacement method on a steady shock. The displacement method also works on unsteady shocks, such as the Sedov blast wave problem. As can be seen in figure 13, the shock-induced displacement versus position behind the shock is no longer approximates a line, but rather a smooth curve. So instead of fitting the displacement to a line, one fits to a curve, typically a polynomial. The position of the shock is still the intercept of the (extrapolated) curve with the horizontal axis: zero shock-induced displacement.

Recall that the Sedov blast wave problem is a unit of energy deposited at the origin of a three-dimensional space filled with cold, unit density, ideal (take $\gamma=5 / 3$ ) gas at rest. The self-similar solution can be obtained numerically integrating a coupled set of ordinary differential equations ${ }^{3}$ or analytically. ${ }^{2}$ The position $r_{s}$ of the shock is

$$
r_{s}=\frac{1}{Q}\left(\frac{E_{x} t^{2}}{\rho_{0}}\right)^{1 / 5}
$$

where $E_{x}=1$ is the deposited energy, $\rho_{0}=1$ is the initial density, and $Q=0.86830699$ is the numerical factor obtained from integration of the self-similar solution's total energy.

With a polynomial fit, the estimation procedure has another parameter: the order of the polynomial. The adequacy of the polynomial order may be checked by inspecting the fit and its residual. Alternatively, one can observe how the estimate depends on the zones selected for the fit, and then choose the polynomial order to minimize that dependence.

The left plot in figure 14 shows how the shock position estimate at $t=0.5$ varies with $n_{z s}$, the number of zones skipped behind the nominal shock front. The number of zones fit, $n_{z f}$, is fixed as $n_{z s}$ varies, but differs for each polynomial order.

Near $n_{z s}=5$, the estimates obtained with a cubic fit vary little. The estimates obtained with a linear fit vary considerably on this scale. The estimates obtained with a quadratic fit vary much less, but the dependence is still noticeable. Considering the limited smoothness of the curves, estimates obtained with a quartic fit are not obviously better than those obtained with a cubic fit. I will thus prefer the cubic fit.

Extrapolation of increasingly higher-order polynomial fits is prone to noise-induced failure. The right plot of figure 14 shows that my fits are well-behaved, and can be reliably extrapolated some distance beyond the fit nodes indicated by the shaded dots. I use only two nodes for the linear fit $\left(n_{z f}=1\right.$ and $\left.n_{z s}=1\right)$. I use 7, 16 and 21 nodes for the quadratic, cubic and quartic fits, respectively. The latter three fits all skip 5 zones behind the nominal shock front $\left(n_{z s}=5\right)$.

Figure 15 shows how the evolution in time of the displacement method's estimates compare with the common estimates of shock position. The maximum derivative and maximum $q$ (artificial viscous stress) methods for estimating shock position can be applied without modification to this blast wave. The half method is adapted like the displacement method, in that the material parameter behind the shock is fit to a quadratic curve and then extrapolated to the vicinity of the captured shock. The half method's estimate of shock position is the location where the linearly interpolated material parameter within the captured shock is half of the extrapolated quadratic fit to the material parameter behind the shock.

The common estimates (from the maximum derivative methods, the maximum $q$ method, and the half methods) all have substantial grid phase oscillations. The peak-to-peak oscillation amplitude of the maximum 


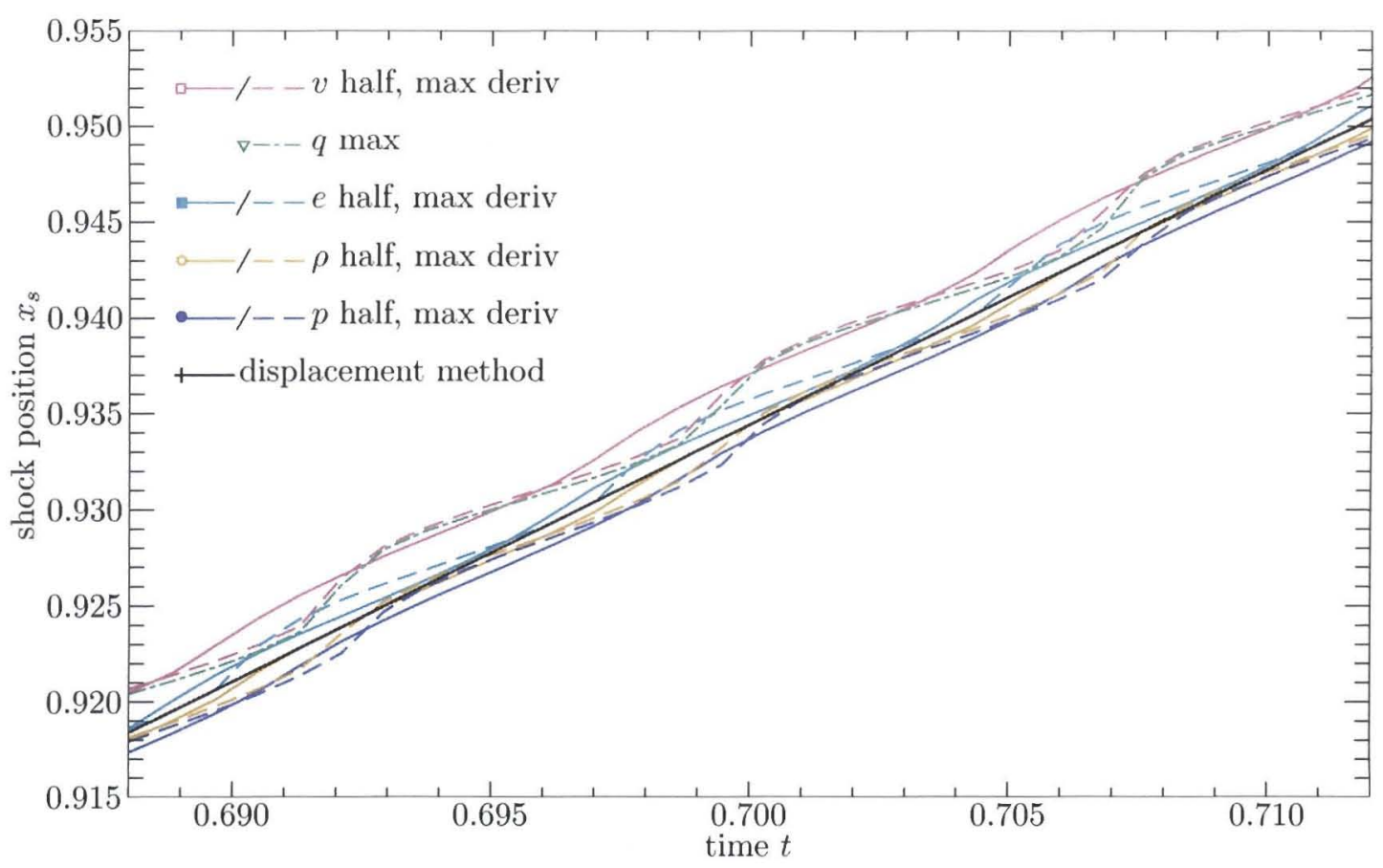

Figure 8. Shock position as a function of time for each time step near $t=0.7$, estimated by the variety of techniques listed in the legend.

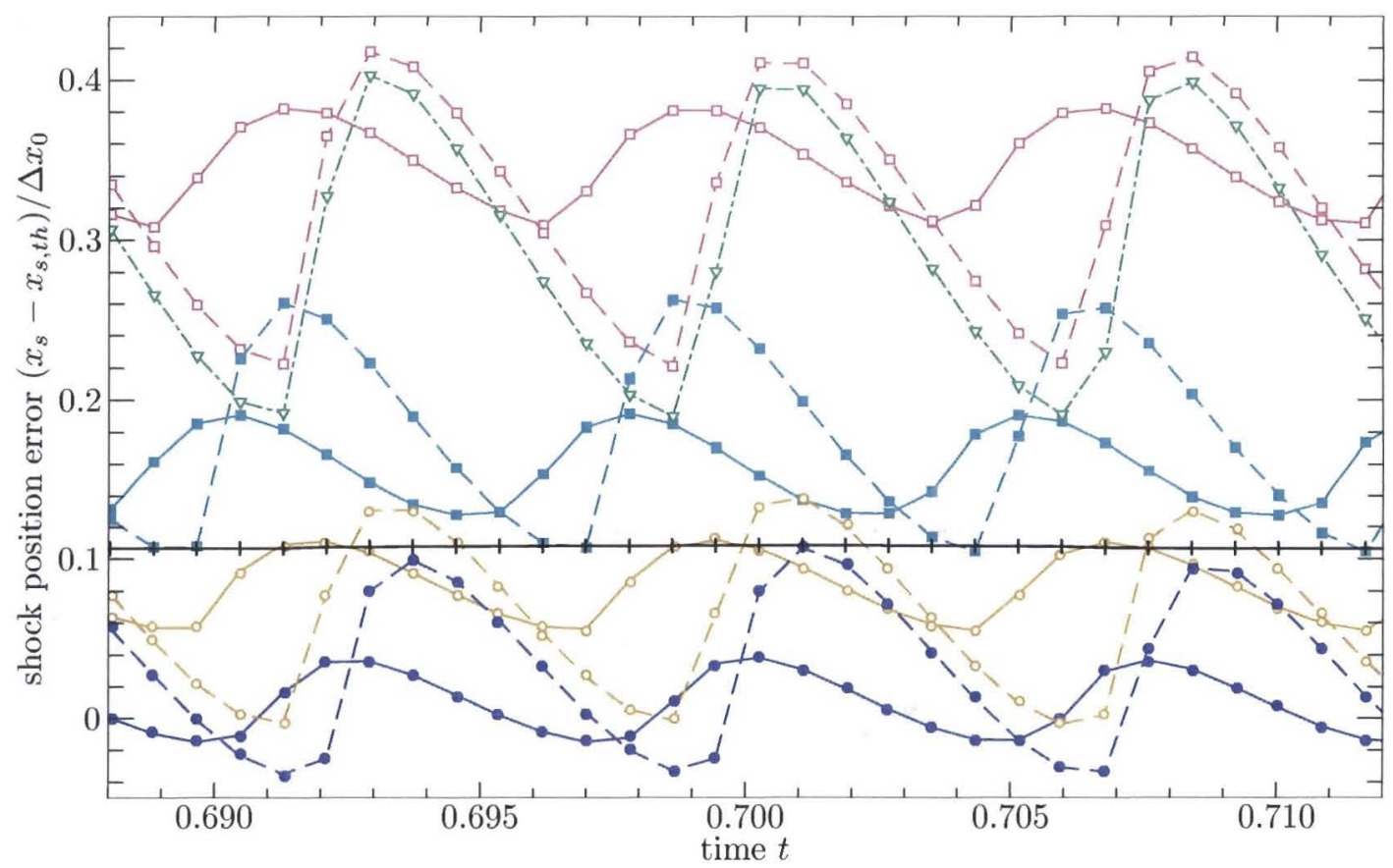

Figure 9. The difference of each estimate from the theoretical shock position $x_{s, t h}$, on a scale relative to the initial zone size $\Delta x_{0}$. The displacement intercept technique (solid black curve) alone is consistent from time step to time step. See previous figure for legend. 


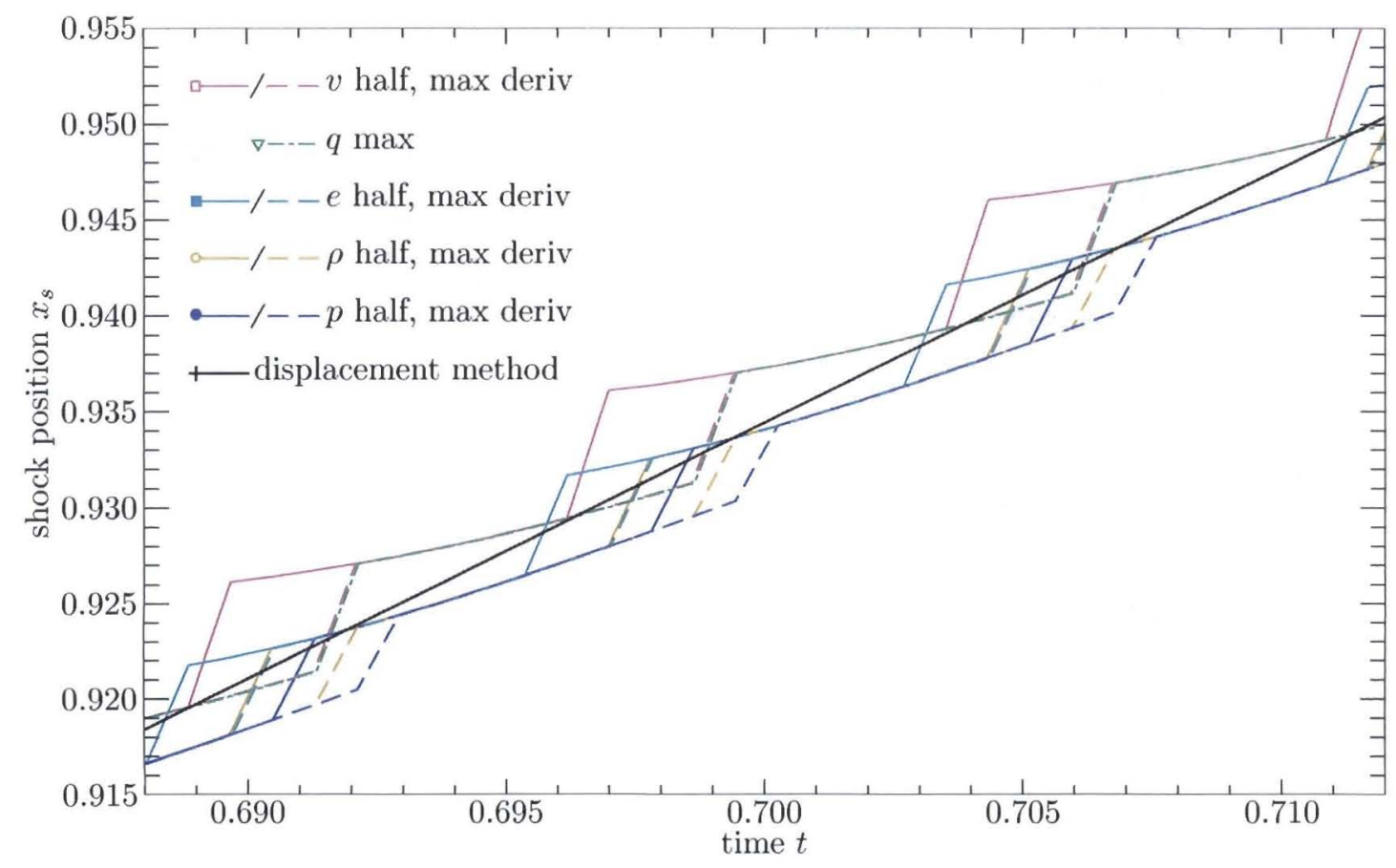

Figure 10. Shock position as a function of time for each time step near $t=0.7$, estimated by the variety of techniques listed in the legend, but with no interpolation for the common estimates. The curves for half $\rho, \max |d v / d x|, \max |d e / d x|$ and $\max q$ are shifted slightly for identification.

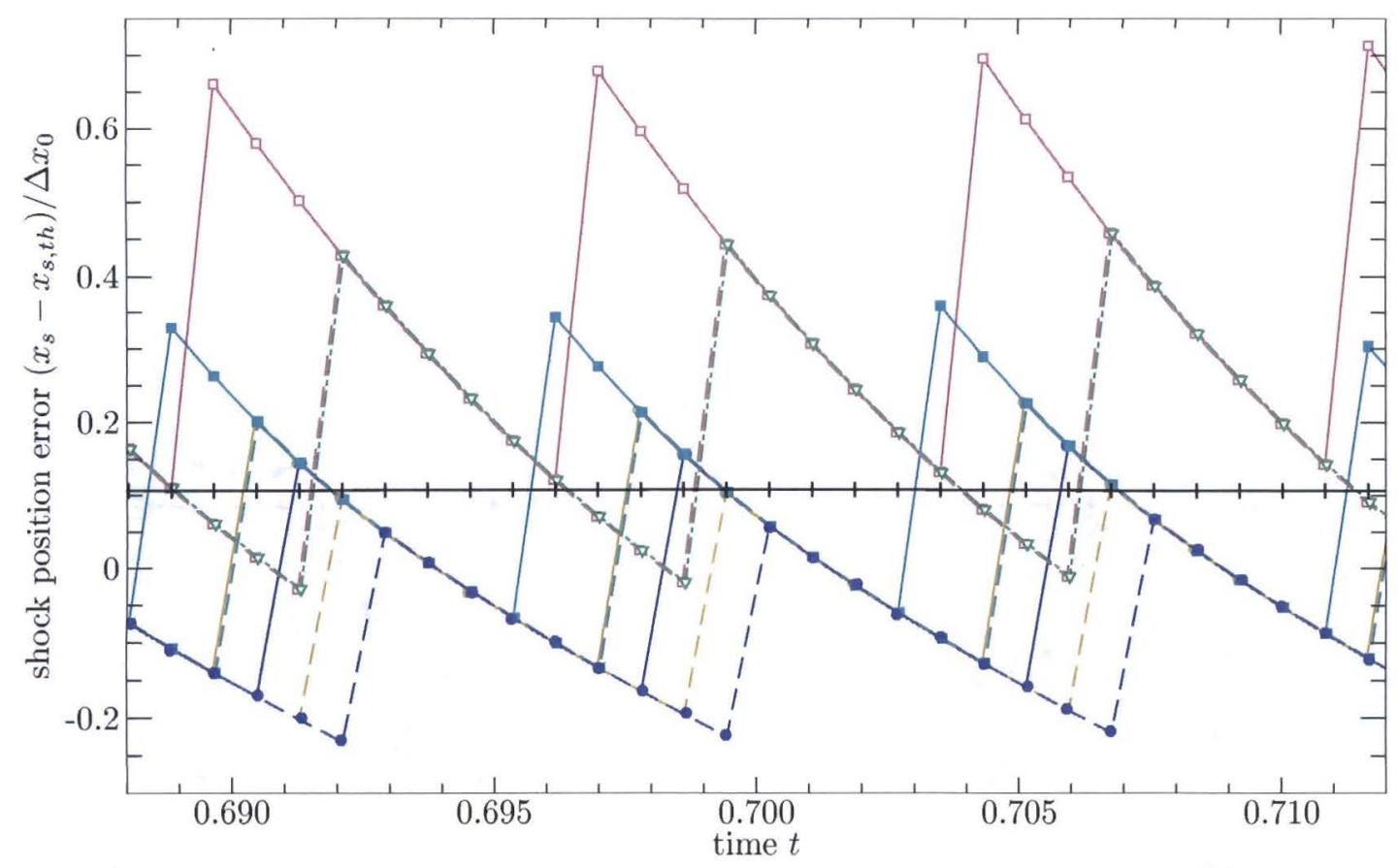

Figure 11. The difference of each estimate (without interpolation) from the theoretical shock position $x_{s, t h}$, on a scale relative to the initial zone size $\Delta x_{0}$. The displacement method (solid black curve) alone is consistent from time step to time step. See previous figure for legend. 


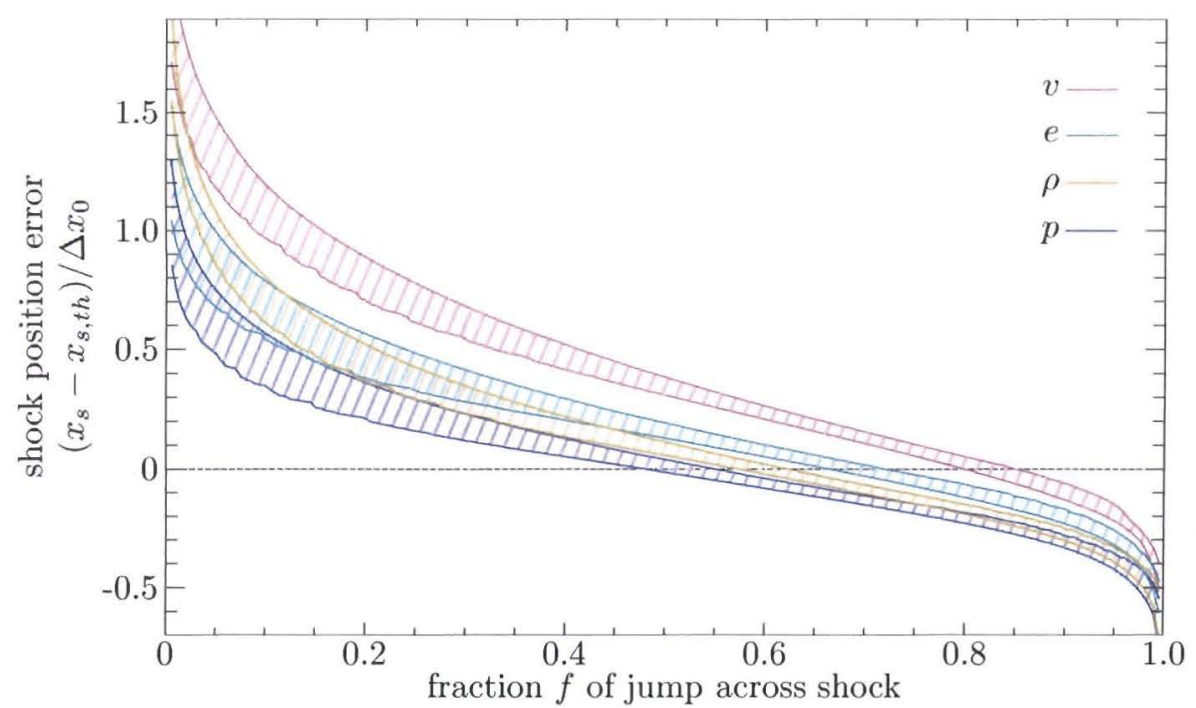

Figure 12. The range (minimum to maximum) of shock position error in the time range $0.688<$ $t<0.712$, as a function of the fraction $f$ of the jump in the material parameter at which the estimate of shock position is located. The material parameter before the shock corresponds to $f=0$, the material parameter after shock corresponds to $f=1$. The half method corresponds to $f=1 / 2$. The reliability of the estimated shock position improves only slightly at higher fraction. No fraction eliminates the variation from time step to time step.

derivative and maximum $q$ methods is about $0.09 \Delta r_{0}$. This oscillation amplitude is larger than the typical $0.027 \Delta r_{0}$ grid phase oscillation observed with the half methods. The estimates based on density $\rho$ have slightly higher oscillation amplitudes: $0.11 \Delta r_{0}$ for the maximum derivative method and $0.05 \Delta r_{0}$ for the half method.

Compared to these oscillation amplitudes, the displacement method's estimates have negligible grid phase oscillations or noise. The displacement method's estimates are thus much more reliable than the common estimates, even for a shock that is as nonsteady as a blast wave.

None of the estimates agree well with theory. The simulated shock positions are ahead of theory by roughly one zone width. This difference is not easily understood. It is not, for example, due to the shock having a head start when the initial energy is deposited "uniformly" throughout the first zone.

\section{Discussion}

I intend to apply the displacement method in my verification studies of impedance matching and shock capturing in a lagrangian hydrodynamics code, where variations of mesh size and artificial viscosity coefficients may change the shock position a fraction of a mesh zone. The method is ideally suited for this application.

However, the method should be useful in other applications where one wants to know how the simulated shock position depends smoothly on continuously variable physical or numerical simulation parameters, or otherwise wants to resolve the simulated shock position to a fraction of the zone size.

The method accommodates a weighted fit just as an average for a material parameter can be weighted. For both the displacement method and for estimates of material velocity, weighting by the mass identified with each node would be typical. Such weighting can reduce the impact of larger errors that may be observed in smaller zones.

In addition to unsteady shocks, the displacement method can be adapted for two-dimensional or threedimensional shock simulations. Instead of fitting to a line (or one-dimensional curve), one fits displacement to a plane (or two-dimensional surface) in two dimensional simulations, or to a hyperplane (or three-dimensional hypersurface) in three dimensional simulations. The intersection of the surface or hypersurface with zero displacement gives the position of the shock. The shock velocity is obtained from the gradient (at zero displacement when the shock is not steady). 

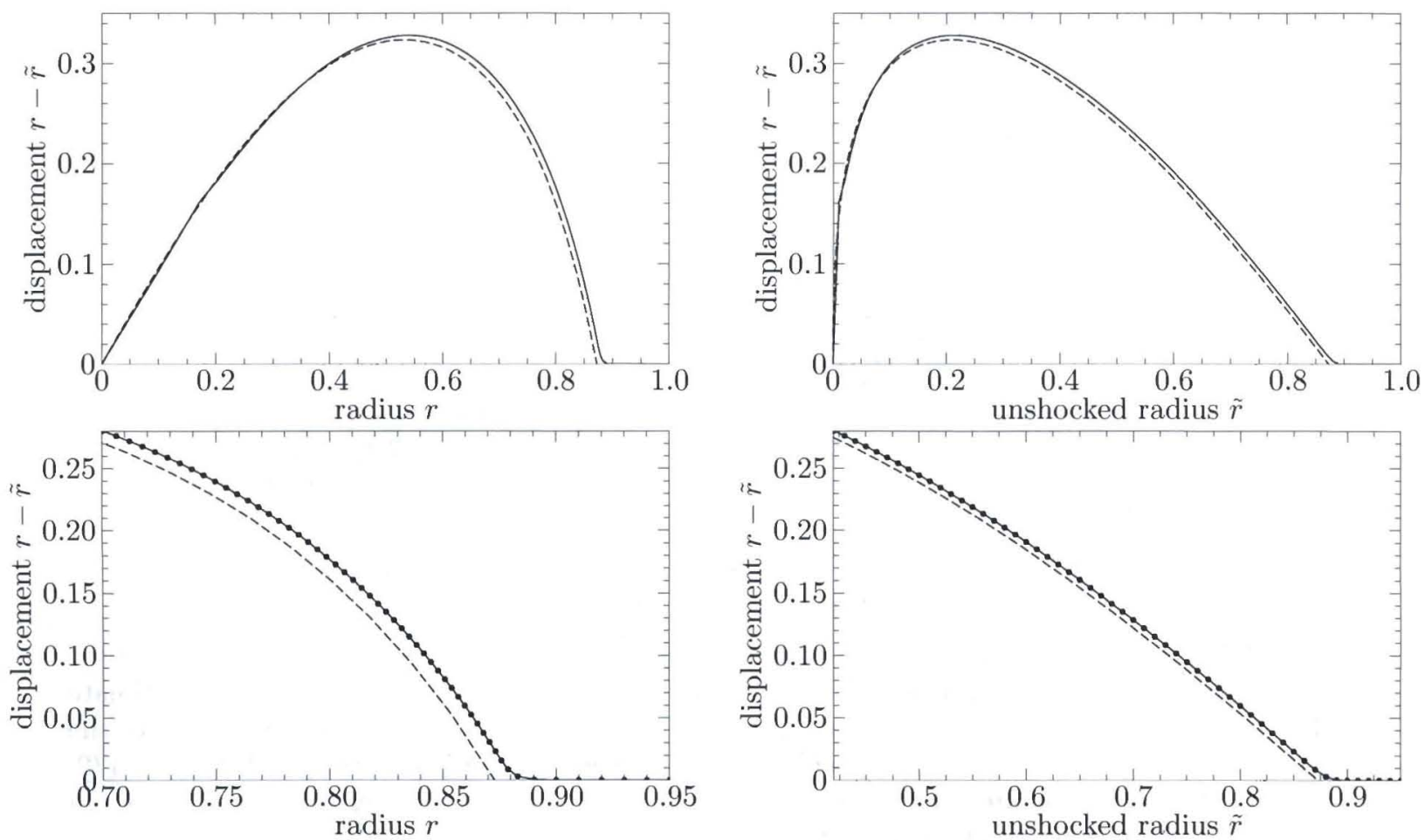

Figure 13. Shock induced displacement $r-\tilde{r}$ versus radius $r$ (left plots) and unshocked radius $\tilde{r}$ (right plots), at $t=0.5$. Top two plots show the entire model. Bottom two plots expand on the shock. Solid curves are the simulated displacement, dashed curves are the theoretical displacement.
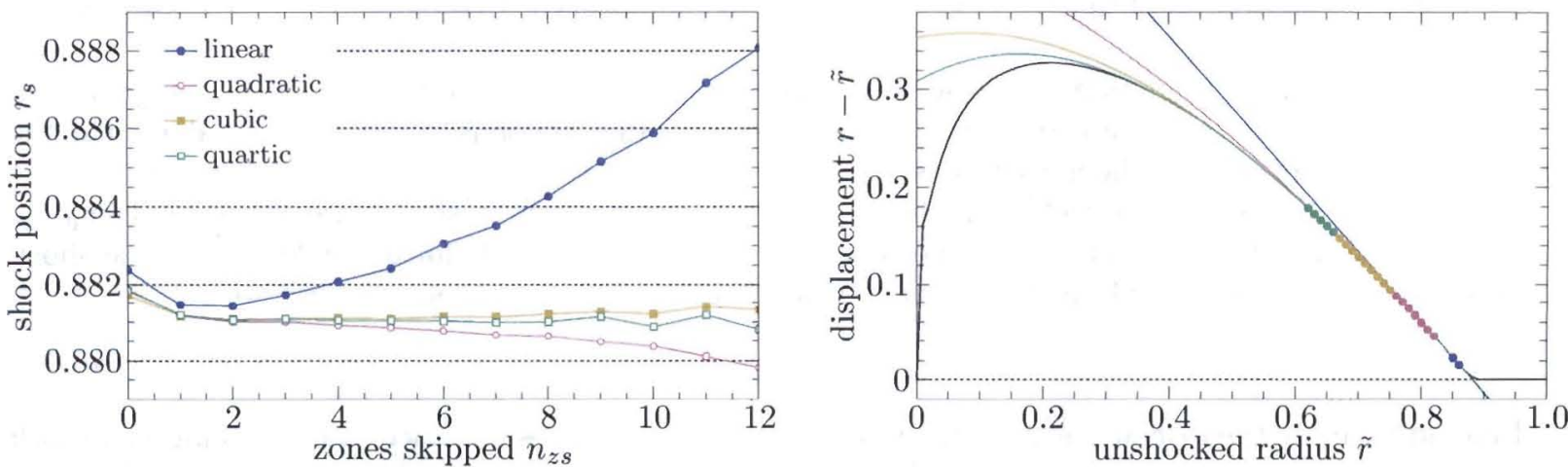

Figure 14. Left plot is the dependence of the shock position estimate on $n_{z s}$, the number of zones skipped behind the nominal shock front, for various orders of the polynomial fit to shock-induced displacement. A cubic fit works well to minimize this dependence. Right plot compares four fits (colored curves, one fit for each polynomial order) with the simulated displacement (black curve). The fits are improving as polynomial order increases. Colored dots are the fit data: 2 nodes for the linear fit, 7, 16 and 21 nodes for the quadratic, cubic and quartic fits, respectively. Nodes for the latter three fits overlap.

The displacement method may be adapted to an eulerian hydrodynamic simulation. In an eulerian simulation, one needs the mass of material passed from one zone to the next. This is the integral over time of the mass flux across a face. Subtracting the mass that would have passed without the shock, one obtains the shock-induced passed mass. Plotting shock-induced passed mass versus position gives a set of points behind the shock to which a curve can be fit, and the intercept of this curve with the horizontal axis (zero shock-induced passed mass) gives the shock position.

The principal limitation of the displacement method is the need to know the unshocked position $\tilde{x}$ of a 


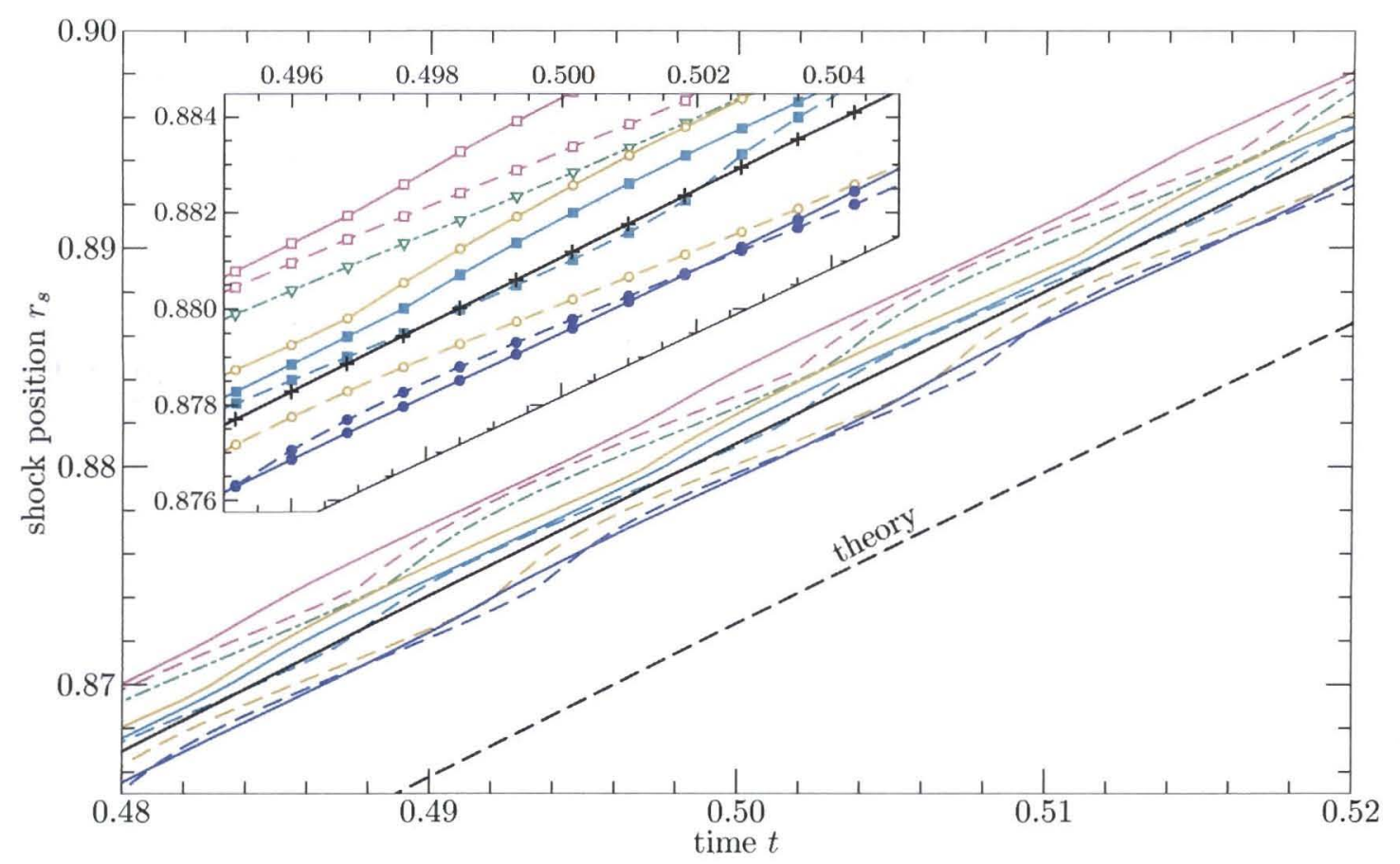

$$
\begin{aligned}
& \square-/--v \text { half } / \text { max derivative } \quad /--\rho \text { half } / \max \text { derivative } \\
& \nabla--q \max \quad-/--p \text { half } / \max \text { derivative } \\
& \text { - / - } e \text { half } / \text { max derivative } \quad+\text { displacement method }
\end{aligned}
$$

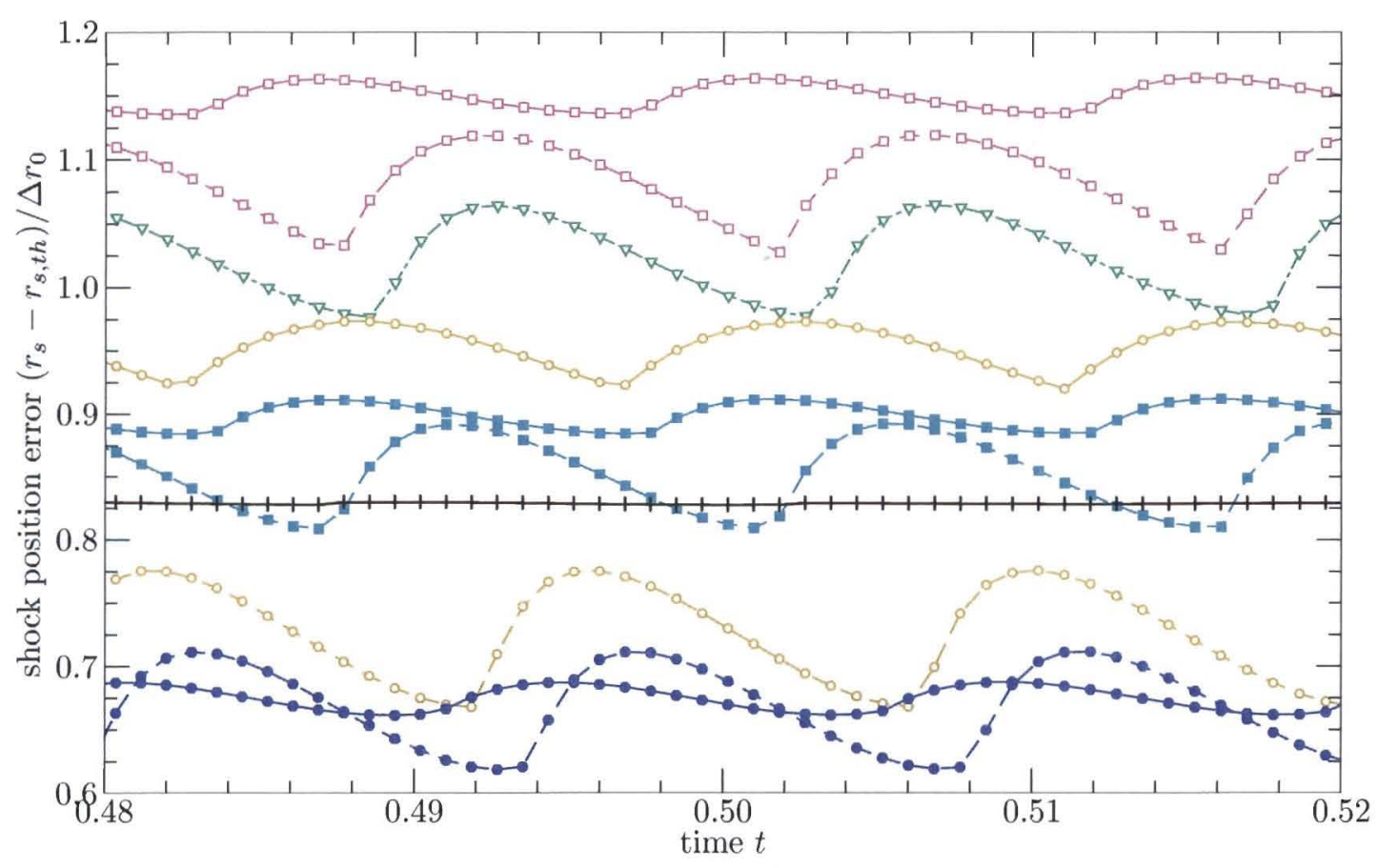

Figure 15. Evolution in time, near $t=0.5$, of the shock position estimates by the maximum derivative methods (dashed curves) and the maximum $q$ method (dot-dash curve). Half method estimates based on quadratic fits to the material parameters are also shown (solid curves), along with the displacement method based on the cubic fit (solid black curve). The bottom plot is the difference of each estimate from the theoretical shock position $r_{s, t h}$, on a scale relative to the initial zone size $\Delta r_{0}$. 
node. The unshocked position is easily obtained when the shock is encountering only undisturbed material. But when the shock of interest is encountering disturbed material, such as from a previous shock, then estimating the unshocked position well can be a chore. In some cases, such as a weak shock in the presence of many comparable or larger waves, or the presence of a material interface just behind the shock, obtaining a sufficiently reliable unshocked position will be intractable. Waves, other shocks or interfaces nearby will similarly degrade or limit estimates of material parameters before or after the shock.

\section{References}

${ }^{1}$ Caramana, E. J., Burton, D. E., Shashkov, M. J., and Whalen, P. P., "The Construction of Compatible Hydrodynamics Algorithms Utilizing Conservation of Total Energy," J. Comp. Phys., Vol. 146, No. 1, 1998, pp. 227262.

${ }^{2}$ Sedov, L. I., Similarity and Dimensional Methods in Mechanics, Academic Press, New York, 1959.

${ }^{3}$ Drake, R. P., High Energy Density Physics, Springer, 2006. 